\title{
Commentary: Advancing completeness and transparency of reporting
}

\author{
T. F. Gressley ${ }^{1 *} \dagger$ and P. L. Ruegg ${ }^{2} \dagger$ \\ ${ }^{1}$ Department of Animal and Food Science, University of Delaware, Newark 19716 \\ ${ }^{2}$ Department of Animal Science, Michigan State University, East Lansing 48864
}

Reporting guidelines are increasingly used by journals to remind authors about important criteria needed to evaluate scientific papers. The Reporting Guidelines for Randomized Controlled Trials for Livestock and Food Safety (REFLECT; https://meridian.cvm.iastate.edu/ reflect/; O'Connor et al., 2010) was published in 2010 to provide guidance to authors on minimum reporting standards in designed experimental studies using livestock. Other guidelines are available that pertain to different types of research that are published in the Journal of Dairy Science. There are many benefits to following such guidelines. These include greater transparency and reproducibility, reduced likelihood of reporting a positive intervention effect, greater potential for future inclusion of published data in meta-analyses, and a greater ability for the reader to identify potential sources of bias. The Journal of Dairy Science welcomes and encourages authors and reviewers to advance the quality of our publications by being aware of and following minimum reporting guidelines.

The developers of REFLECT and other reporting standards have created checklists for authors that remind them of minimum reporting of essential study elements. In this month's invited review by Winder et al. (2019), the authors used the REFLECT checklist to evaluate a random sample of 2017 Journal of Dairy Science publications. They reviewed 120 articles describing 137 controlled trials and assessed reporting of 18 of the 22 REFLECT checklist items. In some cases, they split a single REFLECT item into multiple components, resulting in assessment of 37 specific criteria related to the 18 checklist items. Prevalence of reporting was very high (>90\%) for including descriptions of experimental settings, treatment details, study objectives, outcomes measured, and statistical methodologies. Reporting was moderate to high (47-76\%) for including study unit eligibility, specific hypotheses, use of a washout period

Received April 23, 2019.

Accepted April 23, 2019.

*Corresponding author: gressley@udel.edu

$\dagger$ Gressley and Ruegg are senior and section editor, respectively, of the Health, Behavior, and Well-being section of the Journal of Dairy Science. (when applicable), randomization of treatments, blocking factors, flow of study units through the experiment, the number of study units included in each analysis, and the precision of estimated effect sizes. Reporting of the remaining 23 of the 37 items was low, ranging from 0 to $37 \%$. There is clearly substantial room to improve completeness of reporting, and the review article helps to highlight the specific areas that are in the greatest need of increased reporting.

Many of the REFLECT suggestions mirror concepts taught in classes about design of clinical trials and serve as a reminder to report information that is vital to determining validity of livestock studies. Use of reporting guidelines helps authors remember to include specific criteria during study design and implementation, data analysis, and manuscript preparation. These guidelines also help to standardize the review process by encouraging reviewers to focus on important aspects of the study. The editorial team at the Journal of Dairy Science would like to challenge our authors and reviewers to improve compliance of our publications with the appropriate minimum reporting standards.

The criteria that were least often reported pertained predominantly to some aspects of methodology. Authors can address many of those items with the inclusion of an additional sentence or phrase. For example, adding a sample size justification and calculation, describing eligibility criteria for enrollment, detailing the method used to randomly assign treatments, identifying key roles of individual investigators, describing the presence or absence of blinding, and reporting when no adverse events occurred would all greatly improve transparency of reporting. Some of those details have already been written for the animal care committee overseeing the research, and thus should require minimal effort by the authors to include them in the manuscript. The authors readily know other details that have historically been underreported, and the addition of supplemental text will record that information for the reader. We recommend that researchers review appropriate reporting guidelines before initiating research and follow those guidelines when preparing manuscripts. We also recommend that authors upload a completed checklist during manuscript submission and that the checklist include 
line numbers detailing where each item was addressed. This will help both to ensure that minimum reporting standards were met and to facilitate the review process.

The quality of our journal also depends on our reviewers, and we are grateful to have dedicated reviewers who allocate substantial time and effort to improving Journal of Dairy Science publications. We challenge reviewers to become familiar with reporting standards and to incorporate the appropriate minimum reporting checklist into the review process. When shortcomings are found, we request that reviewers ask authors to include missing information during the revision process.

As representatives of the premier dairy science journal, it is our job to advance reporting standards to enhance reproducibility, transparency, and value. More transparent reporting will benefit both authors and readers by increasing the likelihood for publications to stand the test of time.

\section{REFERENCES}

O'Connor, A.M., J. M. Sargeant, I. A. Gardner, J. S. Dickson, and M. E. Torrence., and consensus meeting participants. 2010. The REFLECT statement: Methods and processes of creating reporting guidelines for randomized controlled trials for livestock and food safety. J. Vet. Intern. Med. 24:57-64. https://doi.org/10.1111/j .1939-1676.2009.0441.x.

Winder, C. B., K. J. Churchill, J. M. Sargeant, S. J. LeBlanc, A. M. O'Connor, and D. L. Renaud. 2019. Invited review: Completeness of reporting of experiments: REFLECTing on a year of animal trials in the Journal of Dairy Science. J. Dairy Sci. 102:4759-4771. https://doi.org/10.3168/jds.2018-15797. 